\title{
The Electrically Short Antenna as a Probe for Measuring Free Electron Densities and Col- lision Frequencies in an Ionized Region ${ }^{1}$
}

\author{
Ronold King, ${ }^{2}$ C. W. Harrison, Jr., ${ }^{3}$ and D. H. Denton, Jr. ${ }^{3}$
}

(February 19, 1961)

\begin{abstract}
If the admittance of a missile, satellite, or drone-aircraft antenna is monitored as the vehicle traverses an ionized region, it is possible to determine the free electron density and the collision frequency of the region if theoretical relations between these quantities are available. In this paper formulas are developed that relate the admittance of an electrically short center-driven dipole or a base-driven monopole when immersed in a conducting dielectric to the effective dielectric constant and conductivity of the medium. From well-known formulas relating these quantities to the free electron density and the collision frequency of an ionized region, these latter may be determined directly from measured admittances. The results obtained when the antenna is treated as a lumped capacitor are considered. It is shown that when the conductivity of the medium is increased to a value that is still quite small, the effect of radiation on the input admittance becomes negligible. The electrically short antenna immersed in sea water is discussed briefly.
\end{abstract}

\section{Introduction}

It has been suggested that electron densities and collision frequencies in the ionosphere may be determined by measuring the impedance of a base-driven electrically short missile antenna, using a lumped-element impedance bridge, as the vehicle traverses the ionized region [Jackson and Kane, $1959 \mathrm{a}$ and b; Haycock and Baker, 1959]. A theoretical relation between the reactance of the $\mathrm{RF}$ probe and the electron density then permits the evaluation of the electron densities from the measured reactances. It is the purpose of this paper to derive an approximate expression for the admittance of a center-driven dipole or of a base-driven probe antenna in a infinite ionized homogeneous medium. The admittance so computed is approximately the same as for an antenna in a medium of finite extent, provided its boundaries are sufficiently far away. It should be mentioned that the impedance of a stub type antenna protruding from a missile differs negligibly from the impedance of the same antenna when mounted on an infinite ground plane, provided the dimensions of the carrying vehicle are not too small in terms of the wavelength.

The formula for the admittance is derived from the integral equation for the distribution of current along the probe by means of a technique that achieves sufficiently high accuracy in the leading terms of an iteration procedure so that higher order terms are not required. The leading terms in the current consist only of linear and parabolic functions with complex coefficients. A method for finding these coefficients is described. No account is taken in the theoretical development of residual charges on the missile [Kraus and Watson, 1958; Jackson and Kane, 1959a; Krassovsky, 1959].

\section{Integral Equation for the Current}

Figure 1 illustrates a cylindrical electric probe of length $2 h$ and radius $a$, center-driven at $z=0$ by an idealized delta function generator of emf $V$. The axis of the antenna coincides with the $z$-axis of a cylindrical system of coordinates $r, \theta, z$. The quasi-one-dimensional theory employed requires the following inequalities to be satisfied:

$$
h \gg a, \quad \beta a=\frac{2 \pi a}{\lambda} \ll 1, \quad \beta=\frac{\omega}{c}
$$

${ }^{1}$ Contribution from the Sandia Laboratory, Albuquerque, N. Mex.

${ }^{2}$ Consultant to the Sandia Corporation and Gordan McKay Professor of Applied Physics, Harvard University.

3 Member of the Scientific Staff, Sandia Corporation. 


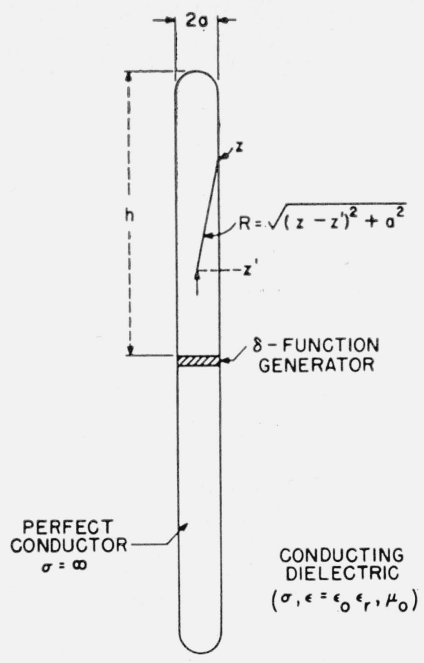

Figure 1. Probe antenna in a conducting dielectric.

where $\lambda$ is the wavelength in the medium. The antenna is assumed to be perfectly conducting and in contact with the dissipative medium characterized by $\mu, \epsilon$, and $\sigma$, the absolute permeability, dielectric constant and conductivity, respectively.

The integral equation for the current along a perfectly conducting symmetrical centerdriven antenna of finite radius immersed in an infinite dissipative medium of arbitrary attenuation is [King and Harrison, 1960]

$$
\frac{4 \pi}{\mu} A_{z}(z)=\int_{-h}^{h} I_{z}\left(z^{\prime}\right) K_{D}\left(z, z^{\prime}\right) d z^{\prime}=j \frac{4 \pi k}{\omega \mu \cos k h}\left[\frac{1}{2} V M_{0 z}+U F_{0 z}\right]
$$

The only restrictions that have been imposed are given by (1).

In $(2), I_{z}\left(z^{\prime}\right)$ is the unknown complex current in the element $d z^{\prime}$ at a distance $z^{\prime}$ from the origin. The distance along the antenna from the origin to the point on the surface of the antenna, where the vector potential $A_{z}(z)$ is computed, is $z$. The kernel $K_{D}\left(z, z^{\prime}\right)$ is

$$
K_{D}\left(z, z^{\prime}\right)=\frac{e}{R}^{-j k R}-{\frac{e}{R_{h}}}^{-j k R_{h}}
$$

where $R=\sqrt{\left(z-z^{\prime}\right)^{2}+a^{2}}, \quad R_{h}=\sqrt{\left(h-z^{\prime}\right)^{2}+a^{2}}$, and $k$ is given by the expression

$$
k=\beta-j \alpha=\omega \sqrt{\mu \epsilon(1-j p)}=\omega \sqrt{\mu \epsilon}[f(p)-j g(p)] ;
$$

$\omega$ is the radian frequency, $f(p)=\cosh \left(1 / 2 \sinh ^{-1} p\right)$ and $g(p)=\sinh \left(1 / 2 \sinh ^{-1} p\right)$, where $p=\sigma / \omega \epsilon$. Tables of the function $f(p)$ and $g(p)$ are a vailable in the literature [King, 1945]. $\omega \mu=k \zeta$, where $\zeta$ is the characteristic impedance of the dissipative medium given by $\zeta=[j \omega \mu /(\sigma+j \omega \epsilon)]^{1 / 2}$ $=\zeta_{e} /[1-j(\alpha / \beta)] . \quad M_{0 z}=\sin k(h-|z|)$ and $F_{0 z}=\cos k z-\cos k h$. The function $U$ is defined by

$$
U=-j \frac{\omega \mu}{4 \pi k} \int_{-h}^{h} I_{z}\left(z^{\prime}\right) \frac{e^{-j k R_{h}}}{R_{h}} d z^{\prime}=-j \frac{\omega}{k} A_{z}(h)
$$

Equation (2) is applicable to an antenna of any length. An RF probe is defined for present purposes as an antenna for which $\beta h \leq 0.3$ radians. For such an electrically short structure, the several functions may be expanded in powers of $k h$ since it is postulated that

$$
\beta h<1, \quad \alpha h<1 .
$$


The kernel $K_{D}\left(z, z^{\prime}\right)$, as given by (3), may be written

$$
K_{D}\left(z, z^{\prime}\right) \approx \frac{1}{R}-\frac{1}{R_{h}}-\frac{k^{2}}{2}\left(R-R_{h}\right)+j \frac{k^{3}}{6}\left(R^{2}-R_{h}^{2}\right) .
$$

Also,

$$
M_{0 z}=\sin k(h-|z|) \approx k(h-|z|)-\frac{k^{3}(h-|z|)^{3}}{6} \approx k h\left(1-\frac{|z|}{h}\right)\left(1-\frac{k^{2} h^{2}}{6}\left[1-\frac{2|z|}{h}+\frac{z^{2}}{h^{2}}\right]\right)
$$

and

$$
F_{0 z}=\cos k z-\cos k h \approx \frac{k^{2} h^{2}}{2}\left(1-\frac{z^{2}}{h^{2}}\right)-\frac{k^{4} h^{4}}{24}\left(1-\frac{z^{4}}{h^{4}}\right) \approx \frac{k^{2} h^{2}}{2}\left(1-\frac{z^{2}}{h^{2}}\right)\left(1-\frac{k^{2} h^{2}}{12}\left[1+\frac{z^{2}}{h^{2}}\right]\right) .
$$

At the driving point $(z=0), M_{0 z}$ and $F_{0 z}$ have the amplitudes $S k h$ and $C k^{2} h^{2} / 2$, in the order named, where $S=1-\left(k^{2} h^{2} / 6\right)$ and $\mathrm{C}=1-\left(k^{2} h^{2} / 12\right)$. It is assumed that $[1-(|z| / h)]$ and $[1-$ $\left(z^{2} / h^{2}\right)$ ] adequately represent the variation, respectively, of $M_{0 z}$ and $F_{0 z}$ along the probe. Accordingly, the simplified representations of $M_{0 z}$ and $F_{0 z}$ are

$$
\begin{aligned}
M_{0 z} & \approx S k h\left(1-\frac{|z|}{h}\right), \\
F_{0 z} & \approx \frac{1}{2} C k^{2} h^{2}\left(1-\frac{z^{2}}{h^{2}}\right) .
\end{aligned}
$$

The function $U$ becomes

$$
U \approx-j \frac{\omega \mu}{4 \pi k} \int_{-h}^{h} I_{z}\left(z^{\prime}\right)\left(\frac{1}{R_{h}}-j k-\frac{k^{2} R_{h}}{2}+j \frac{k^{3} R_{h}^{2}}{6}\right) d z^{\prime}
$$

and

$$
\cos k h \approx\left(1-\frac{k^{2} h^{2}}{2}\right)
$$

In these expansions terms of the order of magnitude $k^{4} h^{4}$ have been neglected.

The final form of the integral equation for the current along a probe antenna in a conducting dielectric is obtained from (2) with (6), (8), and (10). It is

$$
\begin{aligned}
\int_{-h}^{h} I_{z}\left(z^{\prime}\right)\left[\frac{1}{R}-\frac{1}{R_{h}}-\frac{k^{2}}{2}\left(R-R_{h}\right)+j \frac{k^{3}}{6}\left(R^{2}-R_{h}^{2}\right)\right] d z^{\prime} \\
=j \frac{2 \pi k^{2} h}{\omega \mu\left(1-\frac{k^{2} h^{2}}{2}\right)}\left[\operatorname{VS}\left(1-\frac{|z|}{h}\right)+\operatorname{UCkh}\left(1-\frac{z^{2}}{h^{2}}\right)\right] .
\end{aligned}
$$

\section{Some Remarks Pertaining to the Solution of the Free-Space Antenna Equation}

King and Middleton [1946; see also King, 1956, Chapter II] begin the process of obtaining an approximate solution of the free-space antenna equation

$$
\frac{4 \pi}{\mu} A_{z}(z)=\int_{-h}^{h} I_{z}\left(z^{\prime}\right) \frac{e^{-j \beta R}}{R} d z^{\prime}=-j \frac{4 \pi}{\zeta}\left[C_{1} \cos \beta z+\frac{V}{2} \sin \beta|z|\right]
$$

by expressing $I_{z}(z)$ in terms of a reference current $I_{z}\left(z_{0}\right)$ and a distribution function $f(z)$ that is unknown. Let $I_{z}(z)=I_{z}\left(z_{0}\right) f(z)$, so that $I_{z}\left(z^{\prime}\right)=I_{z}\left(z_{0}\right) f\left(z^{\prime}\right)$. Then $I_{z}\left(z^{\prime}\right)=I_{z}(z)\left[f\left(z^{\prime}\right) / f(z)\right]=$ $I_{z}(z) g\left(z, z^{\prime}\right)$. A function $\psi(z)$ is now defined in terms of $g\left(z, z^{\prime}\right)$ by the expression

$$
\psi(z)=\int_{-h}^{h} g\left(z, z^{\prime}\right) \frac{e^{-j \beta R}}{R} d z^{\prime}=\psi\left(z_{1}\right)+\gamma(z),
$$


where $z_{1}$ is a suitable reference point; namely, $z_{1}=0$ when $h \leq \lambda / 4, z_{1}=h-(\lambda / 4)$ when $h>\lambda / 4$. With (13), the integral in (12) may be written in the form

$$
\frac{4 \pi}{\mu} A_{z}(z)=\int_{-h}^{h} I_{z}\left(z^{\prime}\right) \frac{e^{-j \beta R}}{R} d z^{\prime}=I_{z}(z) \psi\left(z_{1}\right)+D(z),
$$

where

$$
D(z)=I_{z}(z) \gamma(z)+\int_{-h}^{h}\left[I_{z}\left(z^{\prime}\right)-I_{z}(z) g\left(z, z^{\prime}\right)\right] \frac{e^{-j \beta R}}{R} d z^{\prime} .
$$

It is evident that (14a) is an identity regardless of whether $g\left(z, z^{\prime}\right)$ is the correct distribution function or not. However, the better the choice of $g\left(z, z^{\prime}\right)$ the smaller is the difference function $D(z)$. If $g\left(z, z^{\prime}\right)$ were precisely correct, the integral in (14b) would vanish, and $\psi(z)$ as defined in (13) would become

$$
\psi(z)=\frac{4 \pi}{\mu} \frac{A_{z}(z)}{I_{z}(z)}
$$

Nevertheless, $D(z)$ in (14a) could not vanish simply because the ratio of the actual vector potential to the actual current is only approximately equal to the constant $\psi\left(z_{1}\right)$. The difference term $D(z)$ is evaluated by successive approximations and its contribution to the expression for $I_{z}(z)$ is contained in the higher-order terms of the solution by iteration.

Owing to the analytical complexity of the higher-order terms in the King-Middleton solution and the fact that its leading trigonometric term includes only a very rough approximation of the susceptive part and no contribution whatsoever to the conductive part of the current, it is desirable for practical purposes to derive an approximate solution for the current in which the leading trigonometric terms are a much better approximation. This may be accomplished if the vector potential and the current are separated into components and these are then properly associated to permit the introduction of several expansion parameters of the type $\psi\left(z_{1}\right)$ in $(13)$. Each of these is the approximately constant ratio of a particular component of vector potential to its associated current. Several integral equations are obtained in this way which may be iterated independently. A study by King [1959] has shown that the sum of only the leading trigonometric terms of these component currents with suitably defined complex coefficients yields the total current and driving point impedance with reasonable accuracy. A precise solution for the distributions of current and charge, and the impedance of an electrically short antenna in free space has been given by King [1952; see also King, 1956 , ch. II, sec. 31], using a method that is not particularly convenient when the antenna is in a dissipative medium.

\section{Solution of the Integral Equation for the Current Along an Electric Probe in an Ionized Medium ${ }^{4}$}

In the King-Middleton antenna theory, the leading reactive term in the complicated series for the current distribution is the familiar sinusoid. Only one $g\left(z, z^{\prime}\right)$ and its associated $\psi(z)$ are used in solving the problem, but several iterations are required to achieve accurate results. On the other hand (11) is arranged in such a way that the leading term consists of two components; one varies as $[1-(|z| / h)]$ and a second varies as $\left[1-\left(z^{2} / h^{2}\right)\right]$. The linear distribution may be considered to be maintained principally and directly by $V$ (which is localized at $z=0)$; the parabolic distribution is induced by the retarded field distributed along the entire antenna and expressed in terms of the equivalent voltage $U$ (as in a receiving antenna

\footnotetext{
4 The procedure employed in this section to solve the integral equation is discussed in somewhat greater detail in Sandia Corp. Tech. Memo. No. 53-60(14), "Approximate solution of the free-space antenna equation based on a determination of the complex coefficients of the leading components and current,' February 1, 1960.
} 
situated in a uniform field). Since the leading terms consist of two components, which are different functions of $z$, these may be expressed separately in terms of the appropriate distribution functions $g_{V}\left(z, z^{\prime}\right)$ and $g_{U}\left(z, z^{\prime}\right)$. These relative distribution functions prove useful in the definition of two expansion functions $\psi_{V}(z)$ and $\psi_{U}(z)$. In order to introduce these functions, it is necessary to separate the total current along the antenna and the vector potential on its surface into component parts, and associate these parts according to their analytical dependence on $z$. The ratio of a particular component of vector potential to its associated current is the constant $\psi_{V}\left(z_{v}\right)$ or $\psi_{U}\left(z_{u}\right)$. Here $z_{v}$ and $z_{u}$ are the values of $z$ where the given part of the total current has its maximum value.

The form of the right-hand side of (11) suggests that it should be possible to approximate the current along an electrically short antenna in a conducting dielectric by

$$
I_{z}\left(z^{\prime}\right)=A M_{0 z^{\prime}}+B F_{0 z^{\prime}}
$$

and evaluate the complex coefficients $A$ and $B$ in such a way that when (16) is substituted into (11), the equation is approximately satisfied. With this substitution,

$$
\begin{aligned}
A \int_{-h}^{h} M_{0 z^{\prime}} K_{D}\left(z, z^{\prime}\right) d z^{\prime}+B \int_{-h}^{h} F_{0 z^{\prime}} K_{D}\left(z, z^{\prime}\right) d z^{\prime} & \\
& =j \frac{2 \pi k^{2} h}{\omega \mu\left(1-\frac{k^{2} h^{2}}{2}\right)}\left\{\operatorname{VS}\left(1-\frac{|z|}{h}\right)+\operatorname{UCkh}\left(1-\frac{z^{2}}{h^{2}}\right)\right\} .
\end{aligned}
$$

The integrals that occur in (17) are

$$
\begin{aligned}
& \int_{-h}^{h} M_{0 z^{\prime}}\left(\frac{1}{R}-\frac{1}{R_{h}}\right) d z^{\prime}, \\
& \int_{-h}^{h} F_{0 \sigma^{\prime}}\left(\frac{1}{R}-\frac{1}{R_{h}}\right) d z^{\prime}, \\
& \int_{-h}^{h} M_{0 z^{\prime}}\left(R^{n}-R_{h}^{n}\right) d z^{\prime}, \\
& \int_{-h}^{h} F_{0 z^{\prime}}\left(R^{n}-R_{h}^{n}\right) d z^{\prime},
\end{aligned}
$$

where $n=1$ or 2 . When these have been evaluated it is found that the leading term in (18a) varies approximately as $M_{0 z}$ and the leading terms in (18b, c, d) vary approximately as $F_{0 z}$.

Equation (17) may now be separated into two parts: One part has an $M_{0 z}$ variation; the other an $F_{0 z}$ variation. Thus,

$$
A \int_{-h}^{h} M_{0 z^{\prime}}\left\{\frac{1}{R}-\frac{1}{R_{h}}\right\} d z^{\prime}=j \frac{2 \pi V S k^{2} h}{\omega \mu\left(1-\frac{k^{2} h^{2}}{2}\right)}\left\{1-\frac{|z|}{h}\right\}
$$

and

$$
\begin{aligned}
A \int_{-h}^{h} M_{0 z^{\prime}}\left\{-\frac{k^{2}}{2}\left(R-R_{h}\right)+j\right. & \left.\frac{k^{3}}{6}\left(R^{2}-R_{h}^{2}\right)\right\} d z^{\prime}+B \int_{-h}^{h} F_{0 z^{\prime}}\left\{\frac{1}{R}-\frac{1}{R_{h}}\right. \\
& \left.-\frac{k^{2}}{2}\left(R-R_{h}\right)+j \frac{k^{3}}{6}\left(R^{2}-R_{h}^{2}\right)\right\} d z^{\prime}=j \frac{2 \pi U C k^{3} h^{2}}{\omega \mu\left(1-\frac{k^{2} h^{2}}{2}\right)}\left\{1-\frac{z^{2}}{h^{2}}\right\} .
\end{aligned}
$$


For convenience let the following notation be used:

$$
\begin{gathered}
\psi_{d 1}=\int_{-h}^{h} g_{v}\left(z, z^{\prime}\right)\left\{\frac{1}{R}-\frac{1}{R_{h}}\right\} d z^{\prime} \\
\psi_{d 2}=\int_{-h}^{h} g_{u v}\left(z, z^{\prime}\right)\left\{-\frac{k^{2}}{2}\left(R-R_{h}\right)+j \frac{k^{3}}{6}\left(R^{2}-R_{h}^{2}\right)\right\} d z^{\prime}
\end{gathered}
$$

and

$$
\psi_{d u}=\int_{-h}^{h} g_{u}\left(z, z^{\prime}\right)\left\{\frac{1}{R}-\frac{1}{R_{h}}-\frac{k^{2}}{2}\left(R-R_{h}\right)+j \frac{k^{3}}{6}\left(R^{2}-R_{h}^{2}\right)\right\}_{z=0} d z^{\prime},
$$

where

$$
\left.\begin{array}{c}
g_{v}\left(z, z^{\prime}\right)=\frac{M_{0 z^{\prime}}}{M_{0 z}}=\frac{\left(1-\frac{\left|z^{\prime}\right|}{h}\right)}{\left(1-\frac{|z|}{h}\right)}, \\
g_{u v}\left(z, z^{\prime}\right)=\frac{M_{0 z^{\prime}}}{F_{0 z}}=\frac{2 S\left(1-\frac{\left|z^{\prime}\right|}{h}\right)}{C k h\left(1-\frac{z^{2}}{h^{2}}\right)}, \\
g_{u}\left(z, z^{\prime}\right)=\frac{F_{0 z^{\prime}}}{F_{0 z}}=\frac{\left(1-\frac{z^{\prime 2}}{h^{2}}\right)}{\left(1-\frac{z^{2}}{h^{2}}\right)} \cdot
\end{array}\right\}
$$

Equations (19) and (20) now become

$$
A \psi_{d 1}=j \frac{2 \pi V k}{\omega \mu\left(1-\frac{k^{2} h^{2}}{2}\right)}
$$

and

$$
A \psi_{d 2}+B \psi_{d u}=j \frac{4 \pi k U}{\omega \mu\left(1-\frac{k^{2} h^{2}}{2}\right)}
$$

respectively. ${ }^{5}$

The substitution of (16) in (9) gives

$$
U=-j \frac{\omega \mu}{4 \pi k}\left\{A \psi_{v}(h)+B \psi_{u}(h)\right\},
$$

where

and

$$
\psi_{v}(h)=S k h \int_{-h}^{h}\left(1-\frac{\left|z^{\prime}\right|}{h}\right)\left\{\frac{1}{R_{h}}-j k-\frac{k^{2} R_{h}}{2}+j \frac{k^{3} R_{h}^{2}}{6}\right\} d z^{\prime}
$$

$$
\psi_{u}(h)=C \frac{k^{2} h^{2}}{2} \int_{-h}^{h}\left(1-\frac{z^{\prime 2}}{h^{2}}\right)\left\{\frac{1}{R_{h}}-j k-\frac{k^{2} R_{h}}{2}+j \frac{k^{3} R_{h}^{2}}{6}\right\} d z^{\prime} .
$$

The use of $U$ from (27) in (26) gives

where

$$
B=A T(h),
$$

$$
T(h)=\frac{\psi_{v}(h)-\psi_{d 2}\left(1-\frac{k^{2} h^{2}}{2}\right)}{\psi_{d u}\left(1-\frac{k^{2} h^{2}}{2}\right)-\psi_{u}(h)} .
$$

\footnotetext{
${ }^{5}$ Equations (19) and (20) are valid for any value of $z$; in particular, they are valid for $z=0$.
} 
Also, from (25),

$$
A=j \frac{2 \pi V k}{\psi_{d 1} \omega \mu\left(1-\frac{k^{2} h^{2}}{2}\right)} .
$$

A tentative expression for the current is obtained when (30) and (32) are substituted in (16) It is

$$
I_{z}(z)=j \frac{2 \pi V S k h}{\psi_{d 1}\left(1-\frac{k^{2} h^{2}}{2}\right) \zeta}\left\{\left(1-\frac{|z|}{h}\right)+T(h) \frac{C}{S} \frac{k h}{2}\left(1-\frac{z^{2}}{h^{2}}\right)\right\}
$$

where use has been made of the relation $\omega \mu=k \zeta$. Readers are reminded that in deriving (33), all difference integrals have been neglected. The parameter $T(h)$ is evaluated from the integrals (21), (22), (23), (28), and (29). It is found that with terms of the order $k^{3} h^{3}$

$$
\begin{aligned}
\psi_{d 1} & \approx 2 \ln \left(\frac{h}{a}\right)-2, \\
\psi_{d 2} & \approx \frac{2 k h}{3}-j \frac{k^{2} h^{2}}{3}-\frac{k^{3} h^{3}}{18}, \\
\psi_{d u} & \approx 2 \ln \left(\frac{2 h}{a}\right)-3+\frac{5}{12} k^{2} h^{2}-j \frac{2}{9} k^{3} h^{3}, \\
\psi_{v}(h) & \approx 2 k h \ln 2-j k^{2} h^{2}-\left(\frac{1}{2}+\frac{\ln 2}{3}\right) k^{3} h^{3}, \\
\psi_{u}(h) & \approx k^{2} h^{2}-j \frac{2}{3} k^{3} h^{3} .
\end{aligned}
$$

An approximate formula for $T(h)$ may be obtained if (34) is substituted into (31) and terms of the order $(k h)^{4}$ are neglected. It is

$$
T(h) \approx \frac{\left(2 \ln 2-\frac{2}{3}\right) k h-j \frac{2}{3} k^{2} h^{2}-k^{3} h^{3}\left(\frac{1}{9}+\frac{\ln 2}{3}\right)}{2 \ln \frac{2 h}{a}-3-k^{2} h^{2}\left(\ln \frac{2 h}{a}-\frac{11}{12}\right)+j \frac{4}{9} k^{3} h^{3}} .
$$

In the derivation of (33) terms of the order $k^{4} h^{4}$ and higher have been neglected in the individual functions. Therefore, when the current is expressed as a series in powers of $k h$, all terms of order higher than $k^{3} h^{3}$ without the common factor $k h$, or higher than $k^{4} h^{4}$ with this factor, must be dropped. As a first step in this expansion and simplification $T(h)$ as given in (35) may be expressed as follows when terms of the order $k^{3} h^{3}$ and higher are neglected (since $T(h)$ occurs only where multiplied by an additional factor $k h)$ :

$$
T(h) \approx \frac{2}{3(\Omega-3)}\left[(3 \ln 2-1) k h-j k^{2} h^{2}\right],
$$

where $\Omega=2 \ln \frac{2 h}{a}$. It follows that including terms of the order $k^{3} h^{3}$,

$$
T(h) \frac{C}{S} \frac{k h}{2}=T(h)\left(1+\frac{k^{2} h^{2}}{12}\right) \frac{k h}{2} \approx \frac{1}{3(\Omega-3)}\left[(3 \ln 2-1) k^{2} h^{2}-j k^{3} h^{3}\right] .
$$

If (37) is inserted in (33) and only terms up to and including the order $k^{3} h^{3}$ are retained exclusive of the common factor, the current (33) is given by

$$
I_{z}(z)=\frac{j 2 \pi k h V}{\zeta \psi_{d 1}}\left\{\left(1+\frac{k^{2} h^{2}}{3}\right)\left(1-\frac{|z|}{h}\right)+\frac{(3 \ln 2-1) k^{2} h^{2}-j k^{3} h^{3}}{3(\Omega-3)}\left(1-\frac{z^{2}}{h^{2}}\right)\right\} .
$$


In (37) and (38) use has been made of the relations $S=\left[1-\left(k^{2} h^{2} / 6\right)\right], C=\left[1-\left(k^{2} h^{2} / 12\right)\right], C / S \approx$ $\left[1+\left(k^{2} h^{2} / 12\right)\right]$, and $\left[1-\left(k^{2} h^{2} / 6\right)\right] /\left[1-\left(k^{2} h^{2} / 2\right)\right] \approx\left[1+\left(k^{2} h^{2} / 3\right)\right]$. The admittance obtained from (38) with $z=0$ is

$$
Y(k)=j \frac{2 \pi k h}{\zeta \psi_{d 1}}\left\{1+\frac{k^{2} h^{2}}{3}\left[1+\frac{(3 \ln 2-1)}{(\Omega-3)}\right]-j \frac{k^{3} h^{3}}{3(\Omega-3)}\right\}
$$

These are the final general formulas for the current in and the admittance of an electrically short center-driven dipole of half-length $h$. The corresponding formulas for a base-driven monopole of length $h$ erected vertically on an infinite ground plane are obtained from (38) and (39) by multiplying the right sides by 2 .

It is now clear why it is necessary to include cubed terms in the small quantity $k h$. For if $\alpha=0$ so that $k=\beta$ and $\zeta=\zeta_{e}$ are real, the only contribution to the real part of $Y(k)$ comes from the term with $k^{3} h^{3}$ as coefficient. Thus, with $Y(k)=G(k)+j B(k)$, the admittance for a dipole in air with $k=\beta_{0}, \zeta=\zeta_{0} \approx 120 \pi$ ohms, is

$$
Y\left(\beta_{0}\right)=\frac{2 \pi \beta_{0}^{4} h^{4}}{3(\Omega-3) \zeta_{0} \psi_{d 1}}+j \frac{2 \pi \beta_{0} h}{\zeta_{0} \psi_{d 1}}\left(1+\frac{\beta_{0}^{2} h^{2} F}{3}\right),
$$

where

$$
F=1+[(3 \ln 2-1) /(\Omega-3)]=1+[1.08 /(\Omega-3)] .
$$

The corresponding expression for the impedance is

$$
Z\left(\beta_{0}\right)=\frac{\zeta_{0} \psi_{d 1}}{6 \pi(\Omega-3)} \frac{\beta_{0}^{2} h^{2}}{\left[1+\frac{\beta_{0}^{2} h^{2}}{3} F\right]^{2}}-j \frac{\zeta_{0} \psi_{d 1}}{2 \pi \beta_{0} h\left[1+\frac{\beta_{0}^{2} h^{2}}{3} F\right]}
$$

For many purposes when $\beta_{0}^{2} h^{2}$ is sufficiently small the correction terms $\beta_{0}^{2} h^{2} F / 3$ may be neglected compared with unity.

\section{Accuracy and Numerical Example When $\alpha=0$}

As a specific numerical example consider an antenna for which $h / a=75, \Omega \approx 10$ so that $\psi_{d 1}=6.635$. With $\zeta_{0} \approx 120 \pi$ ohms it follows that

$$
\begin{gathered}
Y\left(\beta_{0}\right)=\left\{0.120 \beta_{0}^{4} h^{4}+j 2.51 \beta_{\mathrm{c}} h\left[1+0.385 \beta_{0}^{2} h^{2}\right]\right\} \times 10^{-3} \mathrm{mho}, \\
Z\left(\beta_{0}\right)=\frac{19.0 \beta_{0}^{2} h^{2}}{\left[1+0.385 \beta_{0}^{2} h^{2}\right]^{2}}-j \frac{398.1}{\beta_{\mathrm{c}} h\left[1+0.385 \beta_{0}^{2} h^{2}\right]} \text { ohms. }
\end{gathered}
$$

This last formula may be compared with the following more accurate result obtained by another method [King, 1952; see also King, 1956, ch. II, sec. 31]:

$$
Z_{0}=18.3 \beta_{0}^{2} h^{2}\left(1+0.086 \beta_{0}^{2} h^{2}\right)-j \frac{396.0}{\beta_{0} h}\left(1-0.383 \beta_{0}^{2} h^{2}\right) \text { ohms. }
$$

The leading terms in (44) and (45) are clearly in good agreement. Specifically, when $\beta_{0} h=0.3$, the numerical results from (43) to (45) are:

$$
\begin{gathered}
Y\left(\beta_{0}\right)=0.972 \times 10^{-6}+j 0.779 \times 10^{-3} \text { mhos } \\
Z\left(\beta_{0}\right)=1.60-j 1283 \text { ohms, } \\
Z_{0}=1.66-j 1274 \text { ohms }
\end{gathered}
$$


The leading terms alone in (44) and (45) give

$$
\begin{gathered}
Z\left(\beta_{0}\right)=1.71-j 1327 \text { ohms, } \\
Z_{0}=1.65-j 1320 \text { ohms. }
\end{gathered}
$$

It is clear that the corresponding numerical values are in very good agreement. Moreover, the simpler formulas with leading terms only yield results that differ by only about 5 percent from the more accurate values. The accuracy of the formulas (40) and (42) has thus been verified when $\alpha=0$ and $\beta_{0} h \leqq 0.3$.

\section{General Case When $\alpha \neq 0$}

When $k=\beta-j \alpha$ and $\zeta=\zeta_{e}\left(1-j \frac{\alpha}{\beta}\right)$ are complex because $\alpha \neq 0$, the ratio of conductance to susceptance in (39) may differ greatly from its value when $\alpha=0$. This is a consequence of the fact that contributions to the conductance may now come from the leading term with $k h$ as coefficient and not only from the term with $k^{4} h^{4}$ as coefficient as when $k=\beta_{0}$. If the complex values of $k$ and $\zeta$ are inserted in (39) and the real and imaginary parts separated, the result is

where

$$
Y(k)=G(k)+j B(k),
$$

$$
\begin{gathered}
G(k)=\frac{2 \pi}{\zeta_{e} \psi_{d 1}}\left\{\frac{2 \alpha}{\beta}\left[\beta h+\frac{2}{3} \beta^{3} h^{3} F\left(1-\frac{\alpha^{2}}{\beta^{2}}\right)\right]+\frac{\beta^{4} h^{4}}{3(\Omega-3)}\left(1-10 \frac{\alpha^{2}}{\beta^{2}}+5 \frac{\alpha^{4}}{\beta^{4}}\right)\right\}, \\
B(k)=\frac{2 \pi}{\zeta_{e} \psi_{d 1}}\left\{\beta h\left(1-\frac{\alpha^{2}}{\beta^{2}}\right)+\frac{1}{3} \beta^{3} h^{3} F\left(1-6 \frac{\alpha^{2}}{\beta^{2}}+\frac{\alpha^{4}}{\beta^{4}}\right)-\frac{\beta^{4} h^{4}}{3(\Omega-3)} \frac{\alpha}{\beta}\left(5-10 \frac{\alpha^{2}}{\beta^{2}}+\frac{\alpha^{4}}{\beta^{4}}\right)\right\},
\end{gathered}
$$

and $F=1+[(3 \ln 2-1) /(\Omega-3)]$ as in $(41)$.

In many applications $\alpha$ is small compared with $\beta$ so that with (4)

$$
k=\beta\left(1-j \frac{\alpha}{\beta}\right) \approx \omega \sqrt{\mu \epsilon}\left(1-j \frac{p}{2}\right)=\omega \sqrt{\mu \epsilon}\left(1-j \frac{\sigma}{2 \omega \epsilon}\right) .
$$

Let it be assumed that the condition

$$
\left[\frac{2 \alpha}{\beta}\right]^{2} \approx\left[\frac{\sigma}{\omega \epsilon}\right]^{2} \ll 1
$$

is well satisfied and (51) with (52) and (53) reduces to

$$
Y(k)=\frac{2 \pi}{\zeta_{e} \psi_{d 1}}\left\{\frac{2 \alpha}{\beta}\left[\beta h+\frac{2}{3} \beta^{3} h^{3} F\right]+\frac{\beta^{4} h^{4}}{3(\Omega-3)}+j\left[\beta h+\frac{1}{3} \beta^{3} h^{3} F-\frac{5}{3} \frac{\alpha}{\beta} \frac{\beta^{4} h^{4}}{\Omega-3}\right]\right\} .
$$

Note that (55) reduces to (40) when $\alpha=0$.

As a numerical example consider the short dipole, previously considered when in air, immersed in a region of the ionosphere where the number density of the electrons is $N=1.5 \times 10^{11}$ electrons per cubic meter and the collision frequency is $\nu=1.1 \times 10^{5} \mathrm{sec}^{-1}$. At a frequency of $6 \mathrm{Mc} / \mathrm{s}$ the real effective dielectric constant $\epsilon$ and conductivity $\sigma$ are

$$
\begin{gathered}
\epsilon=\epsilon_{j} \epsilon_{r}=\epsilon_{0}\left(1-\frac{N e^{2}}{\epsilon_{0} m\left(\nu^{2}+\omega^{2}\right)}\right)=0.665 \epsilon_{0} \text { farads } / \mathrm{m}, \\
\sigma=\frac{N e^{2} \nu}{m\left(\nu^{2}+\omega^{2}\right)}=3.26 \times 10^{-7} \mathrm{mho} / \mathrm{m},
\end{gathered}
$$


where $e$ is the charge and $m$, the mass of an electron. It follows that $\beta=\beta_{0} \sqrt{\epsilon_{r}}=0.1025$ radians/ $\mathrm{m}, \quad \zeta_{\epsilon}=\zeta_{0} / \sqrt{\epsilon_{r}}=462.3$ ohms, $\alpha=\frac{1}{2} \sigma \zeta_{e}=7.534 \times 10^{-5}$ nepers $/ \mathrm{m}$. Since $\frac{2 \alpha}{\beta}=1.470 \times 10^{-3}$, the simpler formula (55) is applicable. With $\beta_{0} h=0.3, \beta h=0.2447$. When substituted in (55), these values lead to

and

$$
Y(k)=1.12 \times 10^{-6}+j 0.513 \times 10^{-3} \text { mho }
$$

$$
Z(k)=4.26-j 1949 \text { ohms. }
$$

If these results are compared with the corresponding values (46) and (47) for the same antenna in air, it is clear that a significant change in admittance and impedance occurs when the antenna is moved from air into the specified region in the ionosphere.

\section{Determination of the Dielectric Constant and Conductivity With a Short Antenna}

The measured change in the driving-point admittance of an electrically short dipole when it is moved from air into the ionosphere or another conducting dielectric may be used to determine the effective dielectric constant and conductivity of the region. If the susceptance of the antenna when in the ionosphere is $B(k)$, and when in air $B\left(\beta_{0}\right)$, it follows from the imaginary parts of (55) and (40) that

$$
\frac{B(k)}{B\left(\beta_{0}\right)}=\frac{\epsilon_{r} \beta_{0} h+\epsilon_{r}^{2} F \beta_{0}^{3} h^{3} / 3}{\beta_{0} h+F \beta_{0}^{3} h^{3} / 3}=\epsilon_{r}\left\{\frac{1+\epsilon_{r} F \beta_{0}^{2} h^{2} / 3}{1+F \beta_{0}^{2} h^{2} / 3}\right\}
$$

Use has been made of the relation $\zeta_{e}=\zeta_{0} / \sqrt{\epsilon_{r}}$ and $\beta=\beta_{0} \sqrt{\epsilon_{r}}$. Since the terms with $\beta_{0}^{2} h^{2}$ as a coefficient are small, the susceptance ratio differs only slightly from the value $\epsilon_{r}$. If $\boldsymbol{\epsilon}_{r} \approx B(k) / B\left(\beta_{0}\right)$ is taken as the approximate value for substitution in the small correction term, the corrected formula for $\epsilon_{r}$ becomes

$$
\epsilon_{r}=\frac{B(k)}{B\left(\beta_{0}\right)}\left[1+\frac{1}{3} F \beta_{0}^{2} h^{2}\left(1-\frac{B(k)}{B\left(\beta_{0}\right)}\right)\right], \quad \beta_{0}=\frac{\omega_{0}}{c}
$$

where, from $(42), F=1+[(3 \ln 2-1) /(\Omega-3)]$. When $\beta_{0} h$ is sufficiently small, the leading term in (61) may be adequate.

If the admittance of the antenna when it is in the ionosphere is $G(k)+j B(k)$ and the conductance in air is $G\left(\beta_{0}\right)$, it follows from (55) and (40)

$$
\frac{2 \alpha}{\beta}=\frac{\sigma}{\omega \epsilon_{0} \epsilon_{r}}=\frac{G(k)-\epsilon_{r}^{5 / 2} G\left(\beta_{0}\right)}{B(k)\left(1+\frac{1}{3} F \beta^{2} h^{2}\right)} .
$$

As in (61) the small term $\frac{1}{3} F \beta^{2} h^{2}$ may be negligible when $\beta h$ is sufficiently small.

\section{Determination of Electron Density and Collision Frequency}

The formulas (56) and (57) that relate the dielectric constant $\epsilon=\epsilon_{0} \epsilon_{r}$ and the conductivity $\sigma$ of the medium to the electron density $N$ and the collision frequency $\nu$ may be solved for $\nu$ and $N$. Thus,

$$
\begin{gathered}
\nu=\frac{\sigma}{\epsilon_{0}-\sigma} \\
N=\left[\frac{\omega^{2}\left(\epsilon_{0}-\epsilon\right)^{2}+\sigma^{2}}{\epsilon_{0}-\epsilon}\right] \frac{m}{e^{2}} .
\end{gathered}
$$


If $\epsilon=\epsilon_{0} \epsilon_{r}$ is obtained from (61) and $\sigma$ from (62), $\nu$ and $N$ may be evaluated. Thus, both the number density and the collision frequency may be determined from measurements of the impedance of a short dipole successively when immersed in air and in the ionosphere, provided the relations (56) and (57) adequately represent the properties of the latter.

\section{Static Capacitance Formula}

Since it has been assumed that the antenna is electrically short, its admittance may be determined approximately from its lumped capacitance. When the antenna is immersed in air, the approximate susceptance is given by the leading term in (40). That is,

$$
B\left(\beta_{0}\right) \approx \omega C \approx \frac{2 \pi \beta_{0} h}{\zeta_{0} \psi_{d 1}} \approx \omega\left(\frac{2 \pi \epsilon_{0} h}{\psi_{d 1}}\right),
$$

so that with (34), the capacitance is

$$
C=\frac{\pi \epsilon_{0} h}{\ln \frac{h}{a}-1} .
$$

An alternative expression frequently given for the static capacitance is [Küpfmüller, 1932]

$$
C_{0}=\frac{2 \pi \epsilon_{0} h}{2 \ln \frac{2 h}{a}-\ln 12} .
$$

The susceptances and reactances obtained at a frequency of $6 \mathrm{Mc} / \mathrm{s}$ with $\beta_{0} h=0.3$, and $h / a=75$ are, from (67),

from $(66)$,

$$
\omega C_{0}=0.666 \times 10^{-3} \mathrm{mho}, \quad-\frac{1}{\omega C_{0}}=-1502 \mathrm{ohms} ;
$$

$$
\omega C=0.754 \times 10^{-3} \text { mho, } \quad-\frac{1}{\omega C}=-1326 \text { ohms. }
$$

More accurate values according to (46) are

$$
B\left(\beta_{0}\right)=0.779 \times 10^{-3} \text { mho, } \quad X_{0}\left(\beta_{0}\right)=-1283 \text { ohms. }
$$

It is seen that the use of the conventional formula (67) leads to an error of nearly 25 percent in the susceptance of the short antenna in free space.

If the antenna is immersed in a medium with relative dielectric constant $\boldsymbol{\epsilon}_{r}$, the appropriate expressions for the capacitances $C_{\epsilon}$ and $C_{0 \epsilon}$ are simply (66) and (67) multiplied by $\epsilon_{r}$. It follows that

$$
\frac{C_{\epsilon}}{C}=\frac{C_{0 \epsilon}}{C_{0}}=\epsilon_{r} .
$$

This is the same value given by the leading term in (61). Thus, the relatively larger error in susceptance that results from the use of the formula (67) for the static capacitance is canceled out when the ratio (71) is evaluated. It is this ratio that was used, for example, in the measurements reported by Jackson and Kane [1959a]. Note that the above discussion applies when (54) is satisfied.

\section{More Highly Conducting Medium-Plasma and Sea Water}

The generality of the derivation of (39) and (51) has been limited by the conditions (6), $\beta h<1, \alpha h<1$, but the relative magnitudes of $\alpha$ and $\beta$ have not been restricted. In the important special case corresponding to sea water, $\alpha=\beta$, the leading real and imaginary terms in $(51)$ are

$$
Y(k)=\frac{4 \pi}{\zeta_{e} \psi_{d 1}}\left\{\beta h-j \frac{2}{3} F \beta^{3} h^{3}\right\},
$$


where $F$ is defined in (41). The appropriate formulas for $\zeta_{e}$ and $\beta$ are obtained from the defining relations, with $\alpha=\beta, \sigma>>\omega \epsilon$. Thus,

$$
\begin{gathered}
\zeta_{e}=\left[1-j \frac{\alpha}{\beta}\right]\left[\frac{j \omega \mu}{\sigma+j \omega \epsilon}\right]^{1 / 2} \approx\left[\frac{2 \omega \mu}{\sigma}\right]^{1 / 2}, \\
k=\omega[\mu(\epsilon-j \sigma / \omega)]^{1 / 2} \approx[-j \omega \mu \sigma]^{1 / 2}=(1-j)\left[\frac{\omega \mu \sigma}{2}\right]^{1 / 2},
\end{gathered}
$$

so that

$$
\alpha=\beta=\left[\frac{\omega \mu \sigma}{2}\right]^{1 / 2} .
$$

With $Y(k)=G(k)+j B(k)$ as given in $(72)$, it follows that

$$
\begin{gathered}
G(k) \approx \frac{2 \pi h}{\psi_{d 1}} \sigma, \\
B(k)=-\frac{2 \pi h^{3} F}{3 \psi_{d 1}} \omega \mu \sigma^{2}
\end{gathered}
$$

The susceptance of the same antenna in air is

It follows that

$$
B\left(\beta_{0}\right)=\frac{2 \pi}{\zeta_{0} \psi_{d 1}} \beta_{c} h=\frac{2 \pi h}{\psi_{d 1}} \omega \epsilon_{0} .
$$

$$
\frac{\sigma}{\omega \epsilon_{0}}=\frac{G(k)}{B\left(\beta_{0}\right)} \text {. }
$$

This formula may be used to determine $\sigma$ from measured values of the conductance $G(k)$ of the short antenna in the conducting medium with $\alpha=\beta$ and the susceptance $B\left(\beta_{0}\right)$ of the same antenna in air.

Note that the radiation term with the coefficient $\beta^{4} h^{4} / 3(\Omega-3)$, which constitutes the principal contribution to the conductance when the antenna is in air, does not appear in (72) since it is negligible to a high order. Thus, the entire admittance is independent of radiation and the dipole behaves like a pair of electrodes at low frequencies. Moreover, the conductance is very great compared with the susceptance, which is a small quantity of higher order.

The admittance of a dipole immersed in media for which the attenuation constant is in the broad range $0 \leq \alpha \leq \beta$ may be considered in three ranges as follows:

1. $2 \alpha / \beta<<\beta^{3} h^{3} / 3(\Omega-3)<<1$. The dipole is essentially a radiating structure with a conductance that is very small compared with the susceptance.

2. $\beta^{3} h^{3} / 3(\Omega-3)<<2 \alpha / \beta<<1$. The dipole is like a pair of electrodes with negligible radiation. The conductance and susceptance may be comparable in magnutide.

3. $\alpha / \beta \sim 1 ; \beta^{3} h^{3}<<1$. The dipole is like a pair of electrodes with negligible radiation. The conductance is large compared with the susceptance.

Graphs of the input admittance and impedance of an antenna with $\Omega=10, \beta h=0.3$ are shown in figures 2 and 3 as functions of $\alpha / \beta$.

\section{End-Effects and Admittance}

Throughout the analysis it has been assumed that the antenna is center-driven by a socalled delta-function generator which provides a discontinuity in the scalar potential at the center $z=0$ of the antenna. The antenna consists of a perfectly conducting thin tube with adjacent knife edges at $z=0$ along which an electric field $E_{z}(z)=V \delta(z)$ is maintained where $\delta(z)$ is the Dirac delta function. The entire tube with its idealized generator is immersed in the dissipative medium under study. Strictly speaking, the generator is short-circuited by an infinite capacitance and an infinite conductance at the knife edges, so that the current must be 


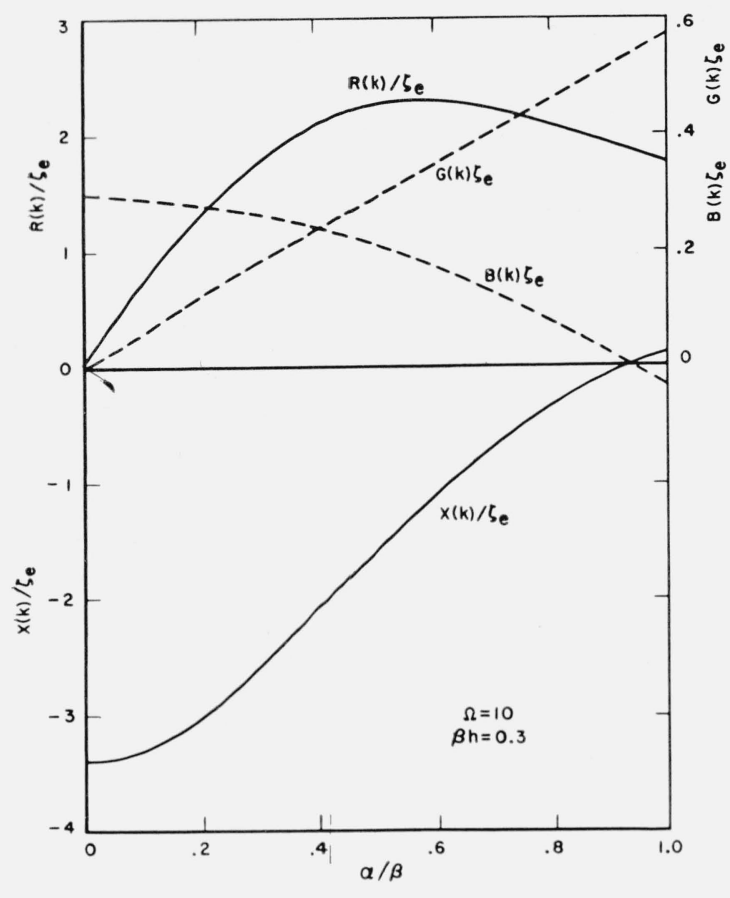

FIgURE 2. Normalized impedance and admittance of short antenna in a dissipative medium.

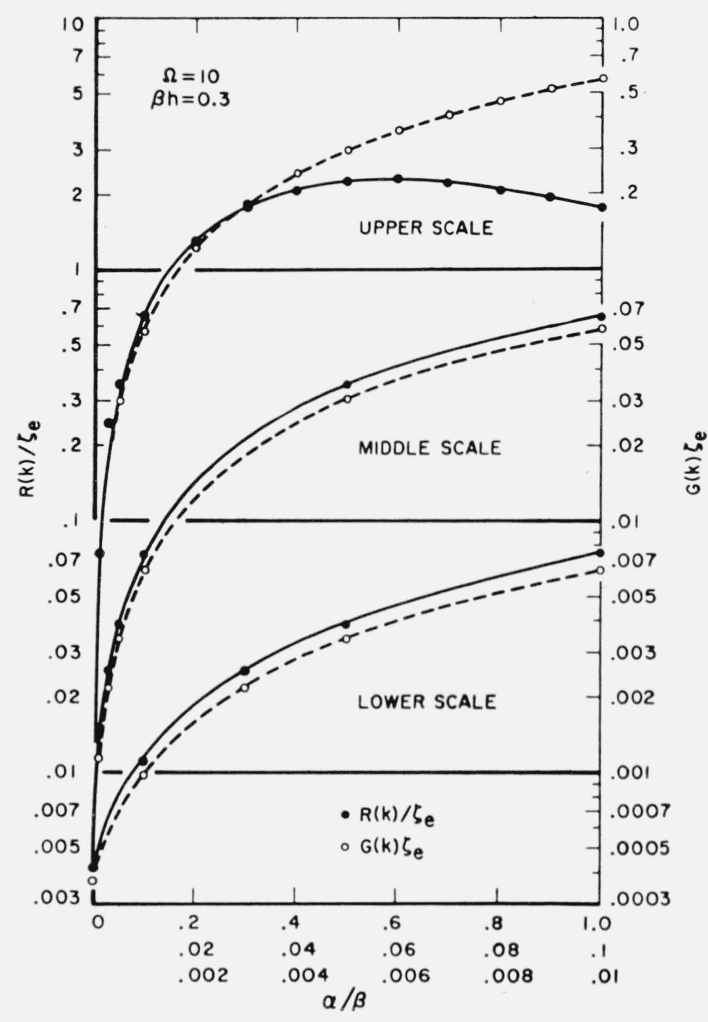

Figure 3. Normalized conductance and resistance of short antenna in a dissipative medium.

infinite. It has been shown by Wu and King [1959] that this large current that is associated with knife edges exists only in an extremely short distance on each side of these edges. Moreover, if the current is approximated by a series of continuous functions, this part of the current can contribute nothing if the solution is not of too high order. Thus, the finite current obtained in this paper is the total current minus the infinite current at the driving point.

An actual antenna used for probing a medium such as the ionosphere may be a dipole center-driven from a two-wire line, or a half dipole base-driven from a coaxial line that pierces a conducting plane. The current entering such an antenna is well approximated by the current supplied by a delta-function generator (minus the infinite knife-edge current) and a small reactive current characteristic of end-effects and coupling effects near the particular junction region between antenna and feeding line [King, 1956, pp. 33-69]. If the line spacing is very small compared with the length of the electrically short antenna, it is necessarily an extremely small fraction of a wavelength. Hence end and coupling effects are negligible.

\section{Conclusion}

Formulas have been derived for the admittance of a perfectly conducting electrically short dipole immersed in an infinite medium that is characterized by a conductivity $\sigma$ and a dielectric constant $\epsilon$ for a range of ratios $\sigma / \omega \epsilon$ that extends from zero to values that are large compared with unity. The application of the formulas to the determination of both $\sigma$ and $\epsilon$ from measured values of the admittance of the antenna when immersed in the medium and when immersed in air has been discussed together with the evaluation of electron densities and collision frequencies of media in which these quantitites may be related directly to $\sigma$ and $\epsilon$. It is to be noted that the more complicated problem of a dipole near the interface between two media has not been treated. 


\section{References}

Haycock, O. C., and K. D. Baker, Determining electron densities in the ionosphere (presented at the 7th Region IRE Conf. and Electronics Exhibit, Univ. N. Mex., May 6-8, 1959).

Jackson, J. E., and J. A. Kane, Measurement of ionospheric electron densities using an RF probe technique, J. Geophys. Research 64, 1074 (1959a).

Jackson, J. E., and J. A. Kane, Breakdown and detuning of transmitting antennas in the ionosphere, Naval Research Laboratory Report No. 5345 (Aug. 24, 1959b).

King, R. W. P., Electromagnetic engineering, appendix 2, pp. 510-518 (McGraw-Hill Book Co., Inc., New York, N.Y., 1945).

King, R. W. P., Theory of electrically short transmitting and receiving antennas, J. Appl. Phys. 23, 1174-1187 (1952).

King, R. W. P., Theory of linear antennas (Harvard Univ. Press, Cambridge, Mass., 1956).

King, R. W. P., Linear arrays: currents, impedances, and fields, I, IRE Trans. on Antennas and Propagation, AP- $\mathbf{7}$, S440-S457 (Special Supplement, Dec. 1959).

King, R. W. P., and C. W. Harrison, Jr., The half-wave cylindrical antenna in a dissipative medium: current and impedance, J. Research NBS 64D, 365 (1960).

King, R. W. P., and D. Middleton, The cylindrical antenna: current and impedance, Quart. Appl. Math. 4, 302 (1946).

Krassovsky, V. I., Exploration of the upper atmosphere with the help of the third Soviet sputnik, Proc. IRE 4\%, 289 (1959)

Kraus, L., and K. M. Watson, Plasma motions induced by satellites in the ionosphere, Physics of Fluids $\mathbf{1}, 480$ (Nov.-Dec. 1958).

Küpfmüller, K., Einführung in die theoretische Elektrotechnik, p. 66 (Julius Springer, Berlin, 1932).

Wu, T. T., and R. W. P. King, Driving point and input admittance of linear antennas, J. Appl. Phys. 30, 74-76 (1959).

\section{Additional References}

Wait, J. R., and L. L. Campbell, The fields of an electric dipole in a semi-infinite conducting medium, J. Geophys. Research 58, 21 (1953).

Wait, J. R., The radiation fields of a horizontal dipole in a semi-infinite dissipative medium, J. Appl. Phys. 24, 958 (1953).

Wait, J. R., and L. L. Campbell, Fields of dipoles in a semi-infinite conducting medium, J. Geophys. Research 58, 21-28, 167-178 (1953).

Wait, J. R., Insulated loop antenna immersed in a conducting medium, J. Research NBS 59, 133 (1957).

Wait, J. R., Radiation from a small loop immersed in a semi-infinite conducting medium, Canad. J. Phys. 37, 672 (1959).

(Paper 65D4-141) 